\title{
New advances in biomimetic surface
}

\author{
REN LuQuan* \\ Key Laboratory of Bionic Engineering, Ministry of Education, Jilin University, Changchun 130022, China
}

Received July 5, 2016; accepted August 30, 2016; published online September 14, 2016

\begin{abstract}
Anti-adhesion is a common phenomenon in living organisms, which is the evolution results to adapt their living surroundings. From the perspective of surface type, there are two typical anti-adhesion mechanisms: micro- and nanosurface structures and liquid-covered surface. Many living organisms possess one or two of these anti-adhesion surfaces in order to achieve superior anti-adhesion, for example, soil animals like mole cricket and earthworm [1]. Carnivorous pitcher plant Nepenthes can capture and digest insects to meet the fundamental nutrients needs. When the insects crawl on its slippery peristome, they could easy-sliding into the picher, known as "aquaplaning". Wong et al. [2] at Harvard University has designed and fabricated slippery surface mimicking this slippery mechanism in Nepenthes, the results of which was published in Nature. However, questions remain about the mechanism underlying its function, especially for the liquid film formation mechanism.

Recently, Chen et al. [3] at Beihang University found the directional water transport phenomenon on the peristome surface, resulting in the complete cover of liquid and long-time holding. They found that this unique water movement phenomenon results from its unique hierarchical structure, which optimized and enhances capillary rise, and preventd backflow in the reverse direction. Specifically, the overlapped duck-billed micro-cavities, scattering along the two-order microgrooves on the peristome surface, have gradient wedge corners and can produce gradient Taylor rise and top-closed gradient Taylor rise, resulting in a continuous filling of the micro-cavities array. Besides, the sharp edge of the microcavity tends to satisfy the condition of Gibbs inequality, leading to water pinning in the reverse direction.

This work further developed the theory of typical capillary rise and the formula of Taylor rise [4]. They found the
\end{abstract}

\footnotetext{
*Corresponding author (email: lqren@jlu.edu.cn)
}

enhancement effect of gradient wedge corner and top-closed wedge corner in capillary rise and deduced the theoretical model for gradient Taylor rise, achieving the theory innovation to perfect the theoretical system of Taylor rise.

They also fabricated artificial peristome with complete morphology characteristics using a bio-replication method by directly employing the nature peristome as the template. By changing the surface wettability of the artificial peristome, they found that hydrophilicity was the essential condition for directional water transport and the superhydrophilic surface possesses the fastest transport speed. This finding gives a guide to design the surface and materials with directional water transport capability.

This work not only discovered a new phenomenon of continuous directional water transport on superwetting surface, but also uncovered the effects of micro- and nanostructures and surface wettability on the directional water transport. They also interpreted the contribution of directional water micro-fluid flow to water long-time holding, i.e., maintaining the slippery function. This directional liquid transport possesses the excellent properties of faster transport, and innovates a new branch of superwetting surface. This new finding can achieve fast continuous directional water transport without external energy source, and the mechanism underlying this phenomenon gives a new insight into designing surface and materials with directional water transport and might find use in applications in anti-adhesion of medical instrument with continuous liquid supply, non-powered self-lubrication in mechanical engineering, MEMS, new energy, and agricultural drip irrigation as well as many other fields in mechanical engineering.

This work is the first paper published in Nature for subject of mechanical engineering in China, and is the new breakthrough in basic scientific research of our mechanical engineering. Bionic engineering can produce initial innova- 
tion and drive the development of conventional mechanical engineering.

1 Ren L. Progress in the bionic study on anti-adhesion and resistance reduction of terrain machines. Sci China Ser E-Tech Sci, 2009, 52: 273-284

2 Wong T S, Kang S H, Tang S K Y, et al. Bioinspired self-repairing slippery surfaces with pressure-stable omniphobicity. Nature, 2011, 477: 443-447

3 Chen $\mathrm{H}$, Zhang $\mathrm{P}$, Zhang $\mathrm{L}$, et al. Continuous directional water transport on the peristome surface of Nepenthes alata. Nature, 2016, 532: 85-89

4 Taylor B. Concerning the ascent of water between two glass planes. Philos Trans Roy Soc Lond, 1710, 27: 538 